\title{
Numerical Simulation of MHD Flow Behavior and Performance in the Disk MHD Generator of Closed Loop Experimental Facility
}

\author{
Alessandro Liberati Non-member (Tokyo Institute of Technology, aleslib@es.titech.ac.jp) \\ Tomoyuki Murakami Member (Tokyo Institute of Technology, murakami@es.titech.ac.jp) \\ Yoshihiro Okuno Member (Tokyo Institute of Technology, yokuno@es.titech.ac.jp) \\ Hiroyuki Yamasaki Member (Tokyo Institute of Technology, yamasaki@es.titech.ac.jp)
}

Keywords: disk MHD generator, closed loop facility, flow behavior, MHD interaction, generator performance

Time dependent r-z two dimensional numerical simulation has been carried out in order to clarify the MHD flow behavior and the performance of the disk MHD generator installed in the new closed loop experimental facility at Tokyo Institute of Technology. The main components of the facility are a compressor, a recuperator, a high temperature gas heater, a seed injection system, a superconducting magnet, a disk shaped MHD generator, a cooler, a gas purification system ${ }^{(1)}$. This paper is focused on the region of the disk MHD generator, shown in Fig. 1, that is here investigated for the first time. In particular, the numerical analysis, that uses a large eddy simulation (LES) model to describe the turbulence of the compressible flow, wants to clarify (1) the behavior of non-MHD flow and MHD flow in the disk MHD generator, and (2) its typical performance in the closed loop experimental facility.

The results show that separation of boundary layers occurs in the downstream part of the generator channel both for non-MHD flow and MHD flow. For the MHD effect, the streamlines tend to widen in the generator channel, with reduction of the thickness of nonMHD flow separation zones, as shown in Fig. 2. Furthermore, the MHD flow presents separation of the boundary layer in the anode region due to the large Lorentz Force.

The performances of the disk MHD generator have been studied using several load resistances and three seed fractions, as shown in Fig. 3. The load resistances with which the maximum enthalpy extraction ratio (E.E.) and isentropic efficiency (I.E.) can be extracted, shifts to lower value as the seed fraction increases. Best performance are obtained for $\mathrm{R}_{\mathrm{L}}=0.2 \Omega$ and $\mathrm{s}_{\mathrm{f}}=8 \times 10^{-4}$. In this case, for thermal input of $0.43 \mathrm{MW}$, an enthalpy extraction ratio of $19 \%$ and isentropic efficiency of $37 \%$ are achieved in the studied disk MHD generator.

The results obtained in this paper will be fruitful to prepare as better as possible and to assure the success of the future MHD closed loop power generation experiment. However, as soon as the

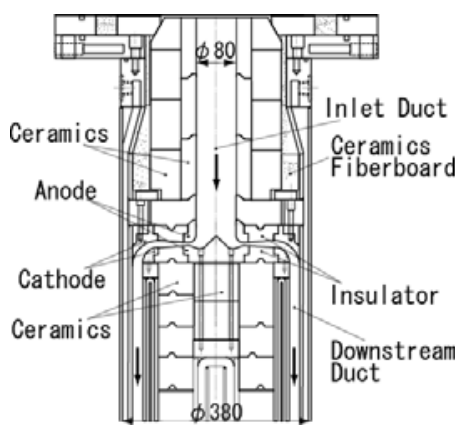

Fig. 1. MHD generator region

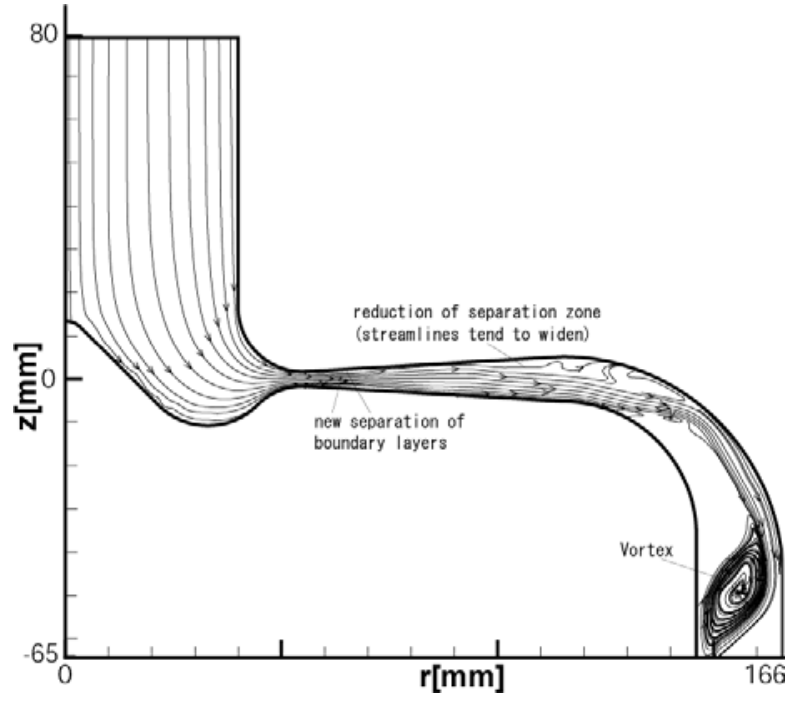

Fig. 2. Velocity streamlines of MHD flow

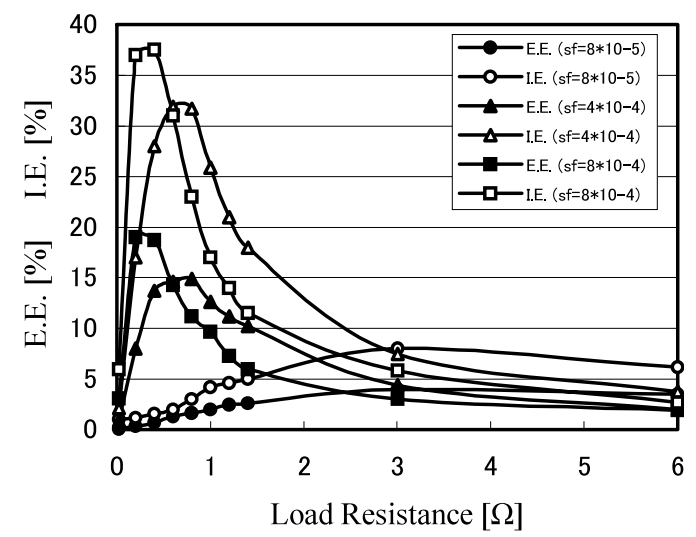

Fig. 3. E.E. and I.E. for different seed fractions against load resistance

experiment will be performed, a new series of simulations will be necessary in order to compare numerical results with experimental ones and to obtain a more reliable description of the problem.

\section{References}

( 1 ) Y. Okuno, T. Murakami, T. Okamura, and H. Yamasaki: "Current Status of Close Loop MHD facility at Tokyo Tech.”, AIAA Paper, 2003-4280 (2003) 


\title{
Numerical Simulation of MHD Flow Behavior and Performance in the Disk MHD Generator of Closed Loop Experimental Facility
}

\author{
Alessandro Liberati* Non-member \\ Tomoyuki Murakami* Member \\ Yoshihiro Okuno* Member \\ Hiroyuki Yamasaki* Member
}

\begin{abstract}
R-z two dimensional numerical simulation with a large eddy simulation (LES) model has been carried out in order to clarify, for the first time, the typical MHD flow behavior and the performance of the disk MHD generator installed in the new closed loop experimental facility at Tokyo Institute of Technology. The results show thick separated flow regions in the generator channel both for non-MHD flow and MHD flow. The separated region influences the MHD interaction because of its low electrical conductivity. The MHD flow streamlines, however, tend to widen in the generator channel, with reduction of thickness of non-MHD flow separation. The typical performance of the generator have been predicted for several load resistances and seed fractions. The study is important to prepare as better as possible and to assure the success of the future MHD power generation experiment.
\end{abstract}

Keywords: disk MHD generator, closed loop facility, flow behavior, MHD interaction, generator performance

\section{Introduction}

A new closed loop experimental facility has been constructed at Tokyo Institute of Technology ${ }^{(1)}$. The facility is a closed cycle MHD electrical power generation system without any conventional gas or steam turbines. The main components of the facility are an oil injection screw type compressor, a recuperator, a high temperature gas heater, a seed injection system, a superconducting magnet, a disk shaped MHD generator, a cooler, a gas purification system. The experimental facility has three important objectives: demonstration of high temperature gas circulation with heat recovery, long time power generation with a disk MHD generator, test of durability of each component. Until now some experiments have been already performed in order to test each component of the facility ${ }^{(2)}$. Also quasi-one dimensional numerical studies have been carried out to suggest the start up operation ${ }^{(3)}$. However, the MHD flow behavior and the performance of the MHD generator installed in the facility have not been yet clarified precisely.

This paper is focused on the MHD-region. Figure 1 shows the cross sectional view of the region. In the channel, the fluid flows downward, axially, in the inlet duct, flows outward, radially, in the MHD generator channel, and flows downward, axially again, in the cylindrical downstream duct. The inlet duct consists of three ceramics layers and watercooled stainless steel vessel. The electrodes of anode and cathode are put on the two disk walls and between them there is an insulator wall made by ceramic. Coaxial inner and outer water-cooled vessels form the cylindrical downstream channel. The main geometry of the disk channel can be changed

\footnotetext{
* Department of Energy Sciences, Tokyo Institute of Technology

4259, Nagatsuta, Midori-ku, Yokohama 226-8502
}

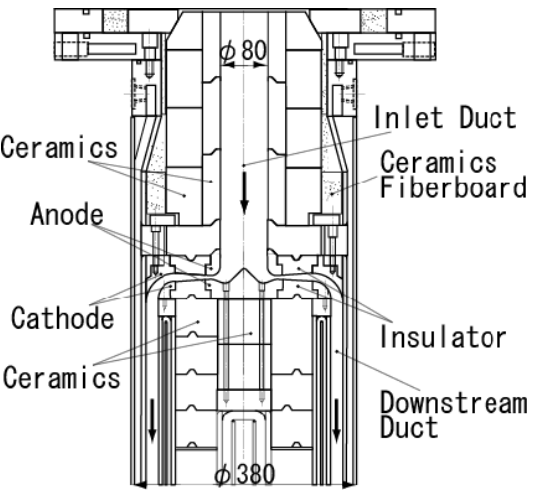

Fig. 1. MHD generator region

varying the height of the throat at the inlet of the divergent channel, but in our study, it is fixed on the basis of the results given by previous numerical simulations ${ }^{(3)}$.

In this paper, the results of an $\mathrm{r}-\mathrm{z}$ two-dimensional numerical simulation for the disk MHD generator region are presented for the first time. In particular, this study wants to clarify (1) the behavior of non-MHD flow and MHD flow in the disk MHD generator, and (2) its typical performance in the closed loop experimental facility. The investigation has not been limited to only the generator channel, but it includes the inlet duct and the downstream $90^{\circ}$-bend diffuser, highlighting the importance of these parts in the design and performance of the generator. Furthermore the paper represents one of the first attempts to use LES in the numerical analysis of a supersonic MHD generator.

\section{Numerical Procedure and Conditions}

2.1 Basic Navier Stokes Equations and LES In order to investigate the non-MHD flow behavior in the channel, 
a large eddy simulation (LES) model has been used to describe the turbulence. In the LES of compressible flow, any field in the flow domain is decomposed into the filtered part, directly resolved at the Grid Scales (GS), and the sub-grid part that accounts for the Sub Grid Scales (SGS) terms which are modeled. In this simulation an implicit top-hat filter has been used with Favre-averaging in order to avoid dealing with double correlation terms including density fluctuation ${ }^{(4)}$. Applying the filter to the Navier Stokes equations, we obtain the equations in filtered form:

- Continuity Equation

$$
\frac{\partial \bar{\rho}}{\partial t}+\frac{\partial\left(\bar{\rho} \tilde{u}_{i}\right)}{\partial x_{i}}=0
$$

- Momentum Equation

$$
\frac{\partial\left(\bar{\rho} \tilde{u}_{i}\right)}{\partial t}+\frac{\partial}{\partial x_{j}}\left(\bar{\rho} \tilde{u}_{i} \tilde{u}_{j}+\bar{p} \delta_{i j}-\bar{\sigma}_{i j}\right)=-\frac{\partial \tau_{i j}}{\partial x_{j}}
$$

\section{- Energy Equation}

$$
\frac{\partial \bar{e}}{\partial t}+\frac{\partial}{\partial x_{j}}\left[(\bar{e}+\bar{p}) \tilde{u}_{j}-\bar{\sigma}_{i j} \tilde{u}_{i}+\tilde{q}_{j}\right]=-\frac{\partial\left(Q_{j}+\frac{1}{2} D_{j}\right)}{\partial x_{j}}
$$

Where $\bar{\sigma}_{i j}$ is the filtered molecular stress tensor and, $\tilde{q}_{j}$ the filtered heat flux. For the static pressure, the filtered equation of state is defined also. In order to evaluate the molecular viscosity and heat conductivity, the Sutherland's formula is used.

2.2 SGS Terms and Basic Assumptions The SGS terms, which have effects on the resolved scales, are modeled using the conventional Smagorinsky model expanded to compressible flow ${ }^{(4)}$. In particular, let's consider the SGS eddy viscosity model,

$$
\tau_{i j}=-\mu_{t}\left(\tilde{u}_{i, j}+\tilde{u}_{j, i}-\frac{2}{3} \delta_{i j} \tilde{u}_{k, k}\right)
$$

With $\mu_{t}$, the turbulent eddy viscosity coefficient as follows:

$$
\mu_{t}=\bar{\rho}\left(C_{s} \Delta\right)^{2}\left[2 \tilde{S}_{i j} \tilde{S}_{i j}-\frac{2}{3}(\nabla \cdot \tilde{u})^{2}+C_{p d} \frac{f_{p d}}{\Delta} \sqrt{(\tilde{c}+|\tilde{u}|)^{2}} \sqrt{(\nabla \cdot \tilde{u})^{2}}\right]^{1 / 2}
$$

where $C_{S}$ is the Smagorinsky coefficient, set to 0.1 in this simulation ${ }^{(5)} . \Delta$ denotes the filter width. In addition a van Driest's wall-damping function is used since the effect of the eddy viscosity is damped toward the wall. $S_{i j}$ is the rate of the strain tensor of the resolvable field. In the expression (5), the term $f_{p d}$ depends by the pressure distribution, detecting the spot where the pressure field is disturbed. The SGS heat flux and SGS turbulent diffusion are modeled on the analogy of the form of the molecular viscosity terms, with Prandtl turbulent number $\operatorname{Pr}=0.9$, as follows:

$$
Q_{j}=-\frac{C_{p} \mu_{t}}{\operatorname{Pr}_{t}} \frac{\partial \tilde{T}}{\partial x_{j}} \quad \cdots D_{j}=-\tau_{i j} \tilde{u}_{j}
$$

It is known that the additional constraints imposed on twodimensional flow change the nature of the turbulence. However, LES is still able to simulate the gross properties of the flow by judicious choice of viscosity and diffusion coefficients ${ }^{(6)}$.
2.3 MHD Equations and Numerical Method In order to investigate the MHD flow and the future performance of the disk MHD generator, MHD equations with two-temperature model, Maxwell equations, together with the equation of state, are used ${ }^{(7)}$.

(1) System for charged particles A non-equilibrium MHD plasma consists of noble gas atoms, noble gas ions, seed atoms, seed ions, and electrons. The governing equations for charged particles are as follows:

- Ionization equation

$$
\begin{aligned}
& \frac{\partial n_{i}^{+}}{\partial t}+\nabla \cdot\left(n_{i}^{+} \vec{u}\right)=\dot{n}_{i}^{+}=k_{f i} n_{e} n_{i}-k_{r} n_{e}^{2} n_{i}^{+} \cdots(7) \\
& n_{e}=\sum_{i} n_{i}^{+} \quad(i=\text { Seed, Noble Gas })
\end{aligned}
$$

- Generalized Ohm's law

$$
\begin{aligned}
& \vec{j}+\frac{\beta}{|\vec{B}|} \vec{j} \times \vec{B}=\sigma(\vec{E}+\vec{u} \times \vec{B}) \\
& \sigma=\frac{e^{2} n_{e}}{m_{e} v_{e}}, \quad \beta=\frac{e B}{m_{e} v_{e}} \cdots \cdots
\end{aligned}
$$

- Conservation of electron energy

$$
\frac{|\vec{j}|^{2}}{\sigma}=3 n_{e} m_{e} k\left(T_{e}-T_{g}\right) \sum_{h} \frac{v_{e h}}{m_{h}}+\sum_{i} \dot{n}_{i}^{+}\left(\frac{3}{2} k T_{e}+\varepsilon_{i}\right)
$$$$
\text { ............. (10) }
$$

( $h=$ Seed, Noble Gas, Ion) $(i=$ Seed, Noble Gas) In the Eq. (6), $k_{f i}$ is the ionization rate coefficient of the $i$ th particle and $k_{r}$ the recombination rate coefficient ${ }^{(8)(9)}$.

(2) System for heavy particles The used equations are the Navier Stokes Eqs. (1), (2), and (3), with the Lorentz force, $\vec{F}_{l}=\vec{j} \times \vec{B}$, added to the right-hand side of the momentum equation (2), and the power density term, $\vec{j} \cdot \vec{E}$, added to the right-hand side of the energy equation (3).

(3) Maxwell equations The MHD approximations of charge neutrality and low magnetic Reynolds number are assumed. Then, Maxwell equations are reduced as follows:

$$
\nabla \times \vec{E}=\overrightarrow{0} \cdots \nabla \cdot \vec{j}=0
$$

The ionization Eq. (7), the continuity Eq. (1), the momentum Eq. (2) and the energy Eq. (3), all of the hyperbolic type, are transformed to a boundary-fitted coordinate, and solved by the CIP method ${ }^{(10)}$ with time step of $\mathrm{dt}=5.0 \mathrm{~ns}$ in order to satisfy CFL condition. The non-linear electron energy Eq. (10) is solved by the bisection method. The substitution of (8) into (11) reduces to an elliptic equation that is discretized with a finite-difference method, and it is solved by the Bi-CGSTAB method ${ }^{(11)}$. The potential function is calculated with the above-mentioned elliptic equation and Maxwell equations.

\subsection{Numerical Region and Boundary Conditions}

Figure 2 shows the numerical region used in the calculation. The inlet duct has a radius of $40 \mathrm{~mm}$. The inner and outer radii of the downstream duct are $146 \mathrm{~mm}$ and $166 \mathrm{~mm}$ respectively. The height of the throat, $\mathrm{h}_{t}$, and the height of the exit of the divergent channel $\mathrm{H}_{\mathrm{e}}$ are $3.3 \mathrm{~mm}$ and $10.3 \mathrm{~mm}$ respectively. The radius at the throat is $55 \mathrm{~mm}$ and the radius at the disk exit is $115 \mathrm{~mm}$, with area ratio A.R. $=6.5$. The anode of the generator is located at $\mathrm{r}=55 \mathrm{~mm} \sim \mathrm{r}=64 \mathrm{~mm}$, the cathode starts from the exit of the divergent channel, 
$\mathrm{r}=115 \mathrm{~mm}$, and it is extended for all the $90^{\circ}$ bend diffuser. The total mesh number is 27206 . The properties of the heavy particles are calculated from the inlet to the outlet of the whole numerical region as in Fig. 2. The electrical values are calculated from the inlet of the anode (anode in) to the exit of the cathode (cathode out). In this part the mesh number is 14458 .

Table 1 shows the working conditions used in the simulation. From the inlet stagnation pressure and temperature, the axial velocity with static pressure and temperature at the inlet are calculated by Rieman's invariant. At the outlet the fixed value of the static pressure is used to solve the characteristics equation.

These inlet and outlet gas conditions can be recognized as typical values in the experimental facility ${ }^{(1)(3)}$, altough the power generation experiment, of course, will be conducted under various gas conditions. For the MHD investigation, cesium seeded argon is used as working gas. Three different values of the seed fraction have been used. The magnetic flux density varies as in the facility from 4.09T, at the upstream edge of the anode, to $4.4 \mathrm{~T}$ at the downstream edge of the cathode. Several load resistances are considered also. Furthermore, in the anode region $(\mathrm{r}=55 \mathrm{~mm} \sim \mathrm{r}=64 \mathrm{~mm})$, the electron temperature (Te) is fixed to $3500 \mathrm{~K}$ and the electron number density is given by Saha equation ${ }^{(7)}$. Te $=3500 \mathrm{~K}$ has been chosen because of the propable condition of weakly ionized gas in the anode region. The value of $\mathrm{Te}$ in this region influences the MHD interaction. The preliminary simulations show that if lower $\mathrm{Te}$ is used, i.e. $\mathrm{Te}=2500 \mathrm{~K}$, this provides no MHD interaction in the generator. If $\mathrm{Te}=5000 \mathrm{~K}$, on the other hand, the main performances are almost the same,

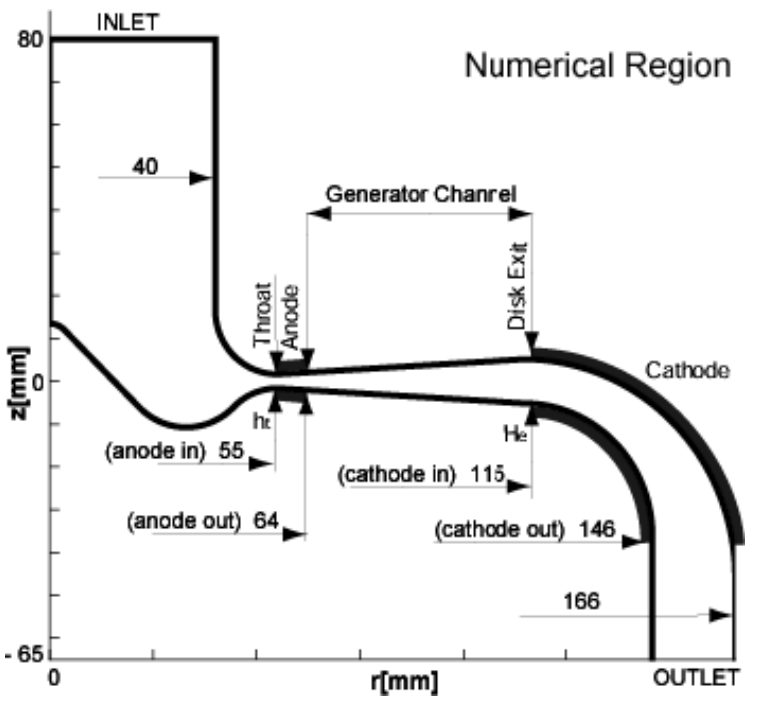

Fig. 2. Numerical region; Total mesh numbers 27206

Table 1. Working conditions

\begin{tabular}{|c|c|}
\hline \multicolumn{2}{|c|}{ Main geometry $\left(\mathrm{h}_{\mathrm{t}}=3.3 \mathrm{~mm}, \mathrm{H}_{\mathrm{e}}=10.3 \mathrm{~mm}\right) \mathrm{AR}=6.5$} \\
\hline Inlet Conditions & $\mathrm{T}_{0}=1800 \mathrm{~K}-\mathrm{P}_{0}=0.40 \mathrm{MPa}$ \\
\hline Outlet Conditions & $\mathrm{P}_{\text {outlet }}=0.065 \mathrm{MPa}$ \\
\hline Seed (Cs) fraction & $8 \times 10^{-5}-4 \times 10^{-4}-8 \times 10^{-4}$ \\
\hline Load Resistance & $0.02-0.2-0.4-0.8-1.0-1.2-1.4-3.0-6.0 \Omega$ \\
\hline Mag. Flux density & $4.09 \sim 4.4 \mathrm{~T}$ \\
\hline
\end{tabular}

whereas the plasma ionization in the generetor is improved.

\section{Results and Discussion}

Until now, the rated high temperature gas has not been circulated in the facility; thus, the numerical and experimental results have not been compared. However, the present numerical model has shown the good agreement for the distribution of static and stagnation pressure in the non-MHD flow ${ }^{(12)}$. This comparison, which was limited to non-MHD case, can confirm the applicability of the model to the present problem.

3.1 Non-MHD Flow Preliminary considerations on non-MHD flow are fundamental in our investigation. In fact, early experiments and calculations have shown that long time (few days) is necessary to the facility to reach the suitable conditions of the argon gas for MHD power generation ${ }^{(2)}$. Then, when MHD starts, the flow in the channel has already its own fluid dynamical behavior, which must be included, as initial conditions, in our numerical simulation, in order to achieve a realistic prediction of the MHD flow and performance of the disk generator. Figure 3 shows the velocity streamlines distribution of non-MHD flow in the MHD generator region. An oblique shock wave is present in the 90 degrees bend diffuser. Across this shock, the turning of the stream occurs with loss of total pressure. Separation of the boundary layer, confirmed in early experiments of the facility ${ }^{(2)}$, occurs on the upper wall, at the exit of the generator channel. The adverse gradient pressure and the presence of the bend-shaped diffuser cause the thick separation zone: the separation point A is shown in Fig. 3. The main flow, however, is supersonic, as shown by the Mach number distribution of Fig. 4. The generator channel could be divided in two parts, depending on the position of the separation point A: in the first part, upstream A, the flow presents an isentropic behavior with thin boundary layers at the wall; in the second part, downstream A, separation of the boundary layers occurs with increase of the static pressure and loss of total pressure in the separated regions. Furthermore, a second separation of the boundary layer occurs on the lower wall of the generator channel. In fact, from separation point $\mathrm{A}$, a weak oblique

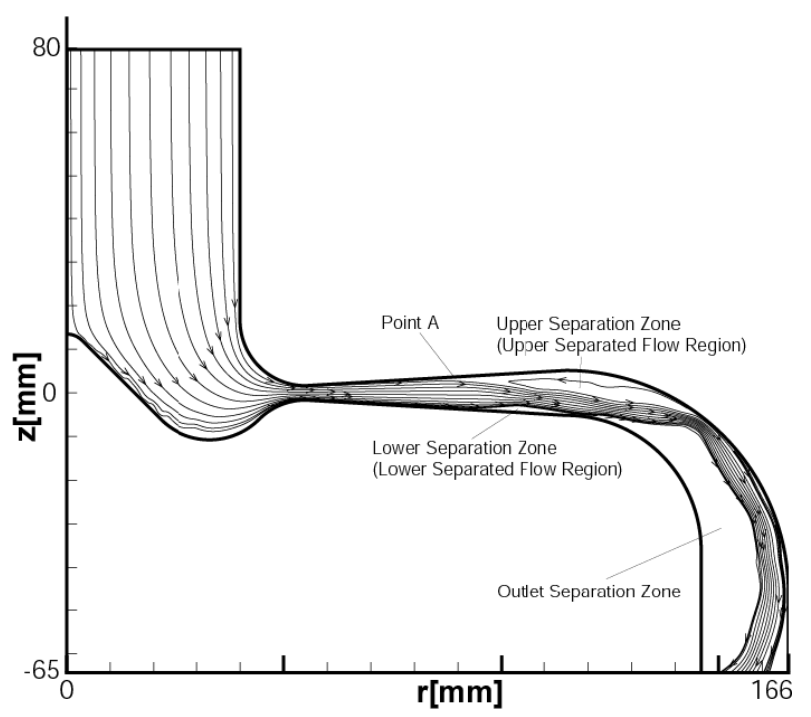

Fig. 3. Velocity streamlines of non-MHD flow 


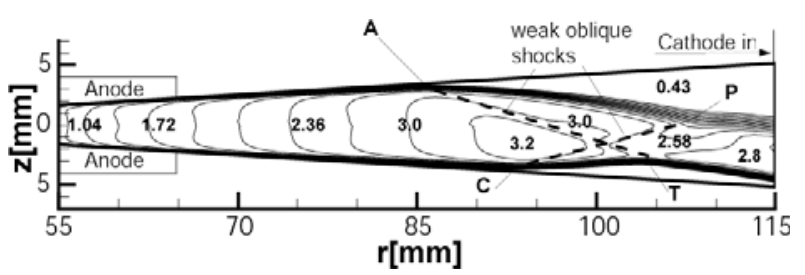

Fig. 4. Mach number distribution in the generator channel

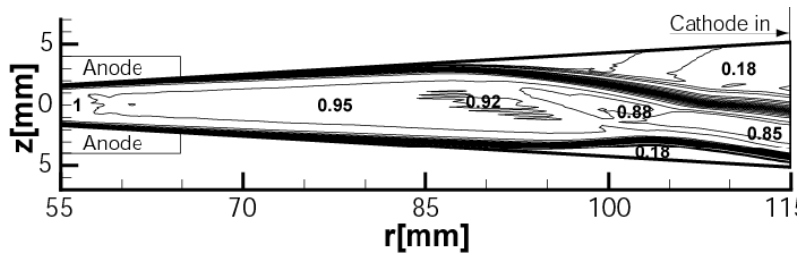

Fig. 5. Normalized total pressure distribution in the generator channel

shock wave is propagated and reflected in the main flow: the reflection of this shock is responsible of the further separated region ${ }^{(13)}$. Qualitatively the dashed line AT of Fig. 4 represents this oblique shock wave. At $\mathrm{T}$, expansions waves are reflected. From point $\mathrm{C}$, compression wavelets coalesce giving another weak oblique shock represented by dashed line CP. At P, expansions wavelets are reflected. In both the separated regions the total pressure is low, as shown in Fig. 5 that depicts the distribution of the normalized total pressure in the generator channel. In the main flow, loss of total pressure are caused by frictional effects, for the high velocity of the flow; after the first separation, the weak shocks propagated in the supersonic flow are responsible for further slight reduction of the total pressure.

3.2 MHD Flow and Performance The typical MHD flow and performances of the generator have been studied using several values of the load resistance, $\mathrm{R}_{\mathrm{L}}$, and three different seed fractions, $\mathrm{s}_{\mathrm{f}}$. Both of these two parameters, in fact, as confirmed by a lot of experiments, strongly influence the behavior of the plasma and the performance of the generator. At the beginning, we will present the performance for all the working conditions used in our study and then we will analyze carefully the particular condition that gives the highest performance of the disk MHD generator.

Figure 6 shows the Hall voltage-Hall current characteristics of the disk MHD generator under the working conditions of Table 1. Figure 7 shows the enthalpy extraction ratio, E.E., (black points) and isentropic efficiency, I.E., (white points) against the load resistances for seed fraction of $8 \times 10^{-5}$ (circle points), $4 \times 10^{-4}$ (triangle points), $8 \times 10^{-4}$ (square points), respectively. The thermal input is $0.43 \mathrm{MW}$. I.E. has been calculated with exit stagnation pressure given by the mean weighted with mass flow rate. This figure shows that the load resistance with which the maximum E.E. and I.E. can be extracted shifts to lower value as the seed fraction increases. This can be ascribed to the increase of the electrical conductivity with seed fraction, especially in the downstream part of the generator channel. For the low seed fraction of $8 \times 10^{-5}$, low E.E. and I.E. are obtained even for high load resistance: low electrical conductivity is present in the disk MHD generator. For the others two seed fraction, relatively high E.E.

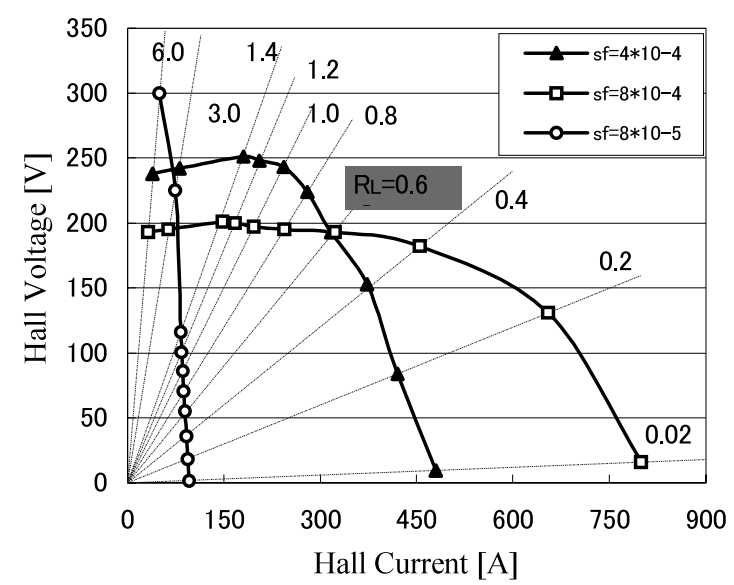

Fig. 6. Hall voltage-Hall current caractheristics for different seed fractions

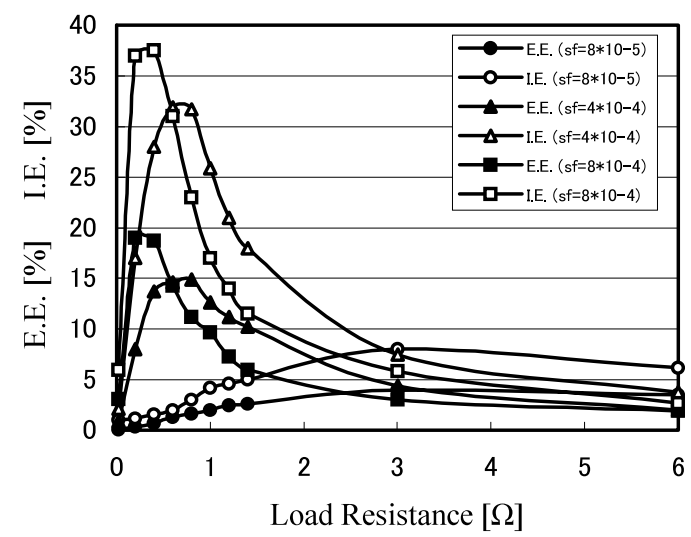

Fig. 7. E.E. and I.E. for different seed fractions against Load Resistance

and I.E. are obtained by this simulation within fixed ranges of load resistance. For $\mathrm{s}_{\mathrm{f}}=4 \times 10^{-4}$, E.E. higher than $13 \%$ and I.E. higher than $25 \%$ are extracted with load resistances between $0.4 \Omega$ and $1.0 \Omega$. For $\mathrm{s}_{\mathrm{f}}=8 \times 10^{-4}$, the figure shows that E.E. higher than $16 \%$ and I.E. higher than $30 \%$ are obtained with load resistances between $0.2 \Omega$ and $0.6 \Omega$. In particular, best performance are achieved with the $\mathrm{s}_{\mathrm{f}}=8 \times 10^{-4}$ and $\mathrm{R}_{\mathrm{L}}=0.2 \Omega$. In this case, Fig. 6 shows that the output voltage is $130 \mathrm{~V}$ and Hall current $605 \mathrm{~A}$, with power output of $84.5 \mathrm{~kW}$ : then, E.E. $=19 \%$ and I.E. $=37 \%$ are obtained by the disk MHD generator. If the seed fraction is increased to $\mathrm{s}_{\mathrm{f}}=1 \times 10^{-3}$, the results are similar to those obtained for $\mathrm{s}_{\mathrm{f}}=8 \times 10^{-4}$, while for $\mathrm{s}_{\mathrm{f}}=4 \times 10^{-3}$, the flow experiences a strong Lorentz force and the velocity decreases, as a result, the performances are deteriorated. Furthermore, numerical results clearly show the existence of a limited range of load resistances that should be found carefully in future experiments in order to produce high power generation in the facility. This is explained as follows. If we use very low value of load resistance, for example the case of $0.02 \Omega$, negative Hall potential appears in the MHD channel owing to the low electrical conductivity in the inlet region, as shown in Fig. 8. The effect of drop of inlet Hall voltage results in the reduction of output Hall voltage and also in the reduction of E.E. and I.E. On the other hand, for high value of load resistance, the Hall voltage tends to be constants with reduction of Hall 


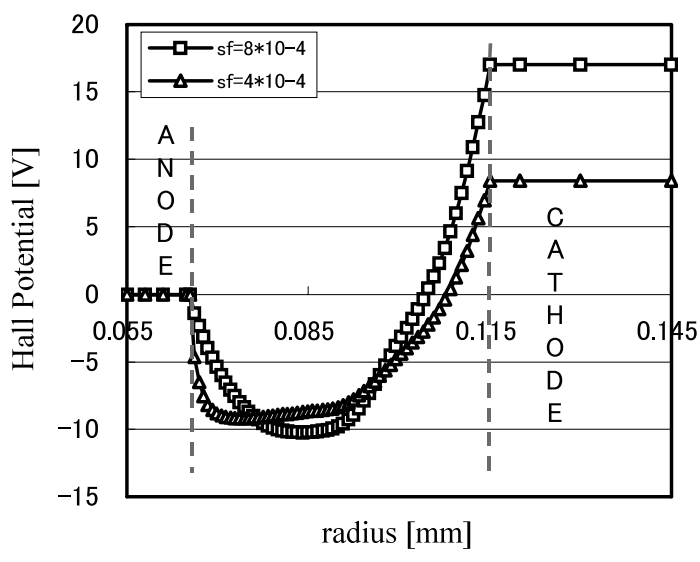

Fig. 8. Hall potential distribution for load resistance $0.02 \Omega$

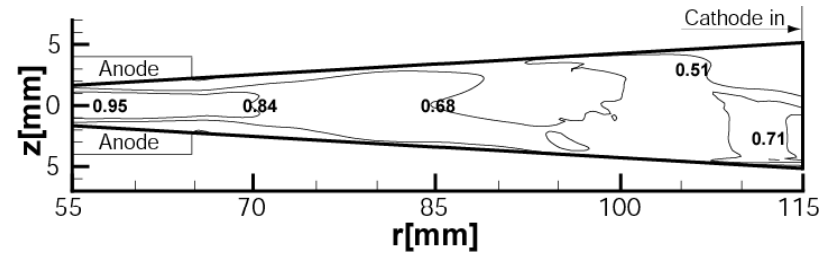

Fig. 9. Mach number distribution in the generator channel for $\mathrm{R}_{\mathrm{L}}=3.0 \Omega$ and $\mathrm{s}_{\mathrm{f}}=8 \times 10^{-4}$

current, and power output, owing to the low gas velocity in the channel, as show in Fig. 9. This figure shows the Mach number distribution in the generator channel for $\mathrm{R}_{\mathrm{L}}=3.0 \Omega$ and $s_{f}=8 \times 10^{-4}$ : the MHD flow is subsonic throughout the entire channel, with consequently reduction of E.E. and I.E. In this sense, as soon as the argon gas, with suitable condition for MHD generation, will be circulated in the facility, with this simulation, setting the real boundaries and several seed fractions, it will be possible to find the range of load resistances, with which perform the MHD experiment in the facility.

It is now useful to do some more considerations on the plasma dynamics in the generator channel, for the optimum condition found above. This further discussion let us to understand the influence of the separation zone on the MHD generation and the mechanism of total pressure loss in the generator. Figure 10 shows the Hall potential distribution in the MHD generator for seed fraction of $\mathrm{s}_{\mathrm{f}}=8 \times 10^{-4}$ and $\mathrm{R}_{\mathrm{L}}=0.2 \Omega$ highest E.E. condition). High Hall field is obtained in the upstream region of the channel. Reduction of the hall field is found in the downstream region. The gradient of the hall potential distribution varies in the generator channel. To understand the reason of this change, let us consider Fig. 11 that represents the Mach number distribution of the MHD flow. Here it is shown that the separation zones of Fig. 4 are strongly reduced by the MHD effect. However, separation of boundary layers still occurs on the upper part of the generator channel. Due to the low electrical conductivity in the separation zone, the effective area of MHD interaction is then reduced in the downstream part of the generator channel, with consequently reduction of the Hall field ${ }^{(14)}$. Our simulations shows that the higher seed fraction, for the increase of electrical conductivity, seems to compensate the reduction of effective area, owing to the reduction of separation zone.

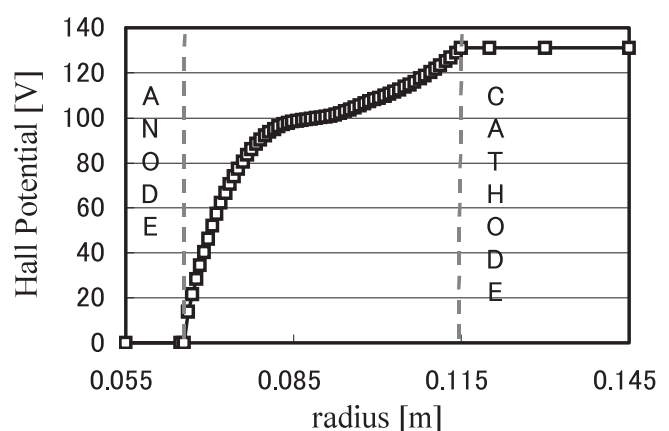

Fig. 10. Hall potential distribution for optimum condition

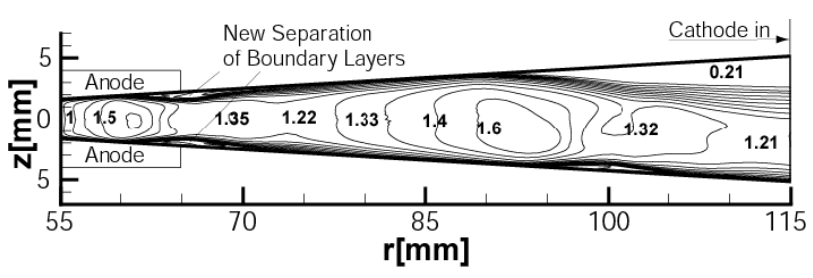

Fig. 11. Mach number distribution in the generator channel for optimum condition

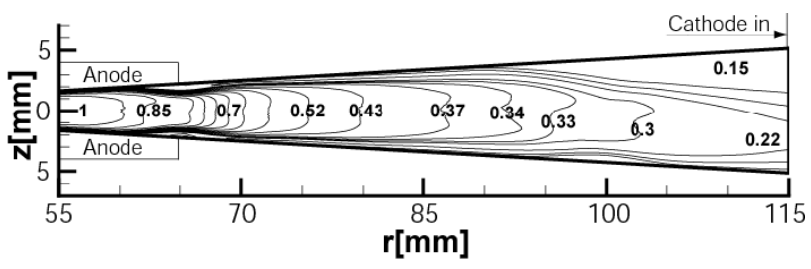

Fig. 12. Normalized total pressure distribution in the generator channel for optimum condition

This lets the Hall field to increase again in the downstream region: for this reason, highest performance can be obtained for high value of seed fraction in this simulation.

Considering again Fig. 11, this shows that a new separation of boundary layers is present across the anode region for the MHD flow. The separation of boundary layers in the anode region has been found also in past simulations ${ }^{(15)}$ and can be explained as follows: in the boundary layer the small Hall parameter due to high gas density and the high conductivity due to non-equilibrium ionization result in a high effective conductivity. Thus, the Faraday current density becomes large in the boundary layer and the corresponding large Lorentz force makes the boundary layer thicker than the situation without magnetic field. In addition, in the downstream region of the anode, a pseudo shock occurs owing to the strong MHD interaction. Also this shock interacts with the boundary layers. These facts are responsible for the separation of the boundary layers near the exit of the anode.

Figure 12 shows the distribution of normalized total pressure in the generator channel for MHD flow. Comparing this distribution with that one in Fig. 5 for non-MHD flow, it is clearly shown that the MHD interaction strongly reduces the total pressure in the main flow of the channel. Furthermore, for the reduction of the gas velocity in the MHD flow, wall frictional loss has minor importance on the mechanism of the total pressure loss. Thus, total pressure is almost consumed by the Lorentz force.

To conclude this discussion, let's consider the MHD-flow 


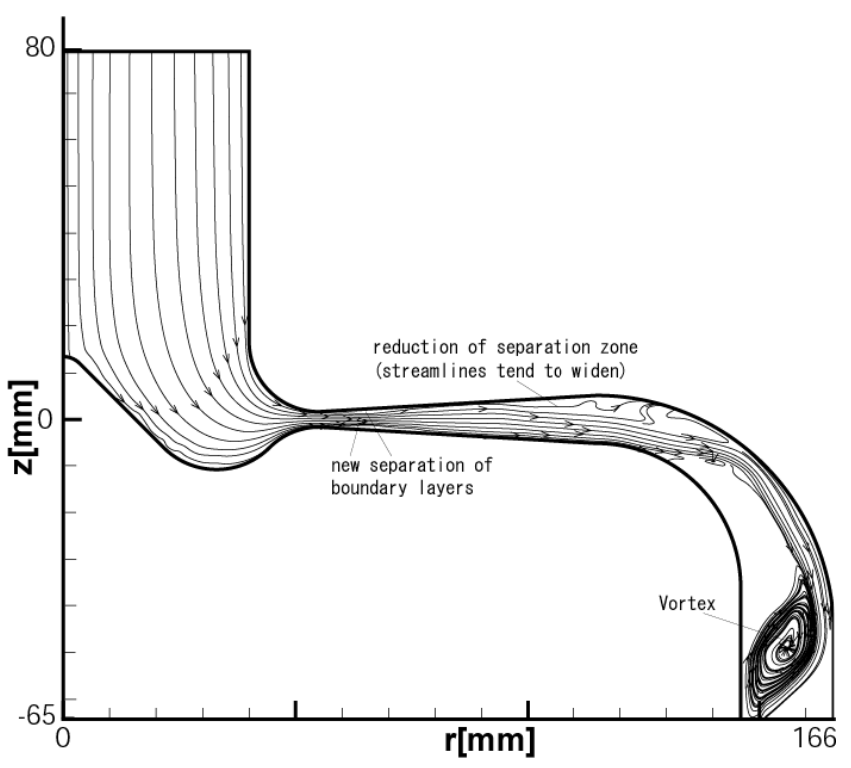

Fig. 13. Velocity streamlines of MHD flow

in the whole numerical region of Fig. 2. Figure 13 shows the velocity streamlines distribution in this region. For the MHD effect, the streamlines tend to widen in the generator channel, with reduction of the thickness of non-MHD flow separation zones. Separations of the boundary layer occurs in anode region. In the $90^{\circ}$ bend diffuser an oblique shock wave is still present to let the turning of the stream. An eddy vortex appears in the outlet cylindrical duct. This can be explained with the reduction of the inertial forces at the exit of the $90^{\circ}$ bend diffuser and the influence of adverse gradient pressure. This vortex seems not to affect the performance of the geneator. However, for higher value of outlet pressure, the vortex might appear in the divergent channel, deteriorating the performance.

Finally, the fluid behavior as well as generator performance can strongly depend on the gas conditions, for example, the pressure ratio $\left(\mathrm{P}_{\mathrm{o}} / \mathrm{P}_{\text {outlet }}\right)$. These gas conditions are controllable in the facility. Thus investigation for other gas conditions is needed; this will be presented in a future work.

\section{Conclusions}

Time dependent r-z two-dimensional large eddy simulation (LES) has been carried out in order to clarify for the first time the MHD flow behavior and the performance of the disk MHD generator of the closed loop experimental facility at Tokyo Institute of Technology. The investigation has not been limited to only the MHD generator channel, but it includes generator's inlet duct and downstream $90^{\circ}$-bend diffuser.

The results show that separation of boundary layers occurs in the downstream part of the generator channel both for non-MHD flow and MHD flow. For the MHD effect, however, the streamlines tend to widen in the generator channel, with reduction of the thickness of non-MHD flow separation zones. Furthermore, the MHD flow presents separation of the boundary layer in the anode region due to the large Lorentz Force. The performances of the disk MHD generator have been studied using several load resistances and three seed fractions. The load resistances with which the maximum enthalpy extraction ratio and isentropic efficiency can be extracted, shifts to lower value as the seed fraction increases. Best performance are obtained for $\mathrm{R}_{\mathrm{L}}=0.2 \Omega$ and $\mathrm{s}_{\mathrm{f}}=8 \times 10^{-4}$. In this case, for thermal input of $0.43 \mathrm{MW}$, enthalpy extraction ratio of $19 \%$ and isentropic efficiency of $37 \%$ are achieved in the studied disk MHD generator. All these results will be fruitful to prepare as better as possible and to assure the success of the future MHD closed loop power generation experiment. However, as soon as the experiment will be performed, a new series of simulations will be necessary in order to compare numerical results with experimental ones and to obtain a more reliable description of the problem.

(Manuscript received Nov. 25, 2005, revised March 2, 2006)

\section{References}

( 1 ) Y. Okuno, T. Murakami, T. Okamura, and H. Yamasaki: "Current Status of Close Loop MHD facility at Tokyo Tech.”, AIAA Paper, 2003-4280 (2003)

( 2 ) T. Murakami, Y. Okuno, and H. Yamasaki: "Experimental results of fluid dynamical behavior and thermal performance of closed loop facility", Proc. $15^{\text {th }}$ Int. Conf. on MHD Energy Conv., Vol.1, pp.45-51, Moscow (2005)

( 3 ) H. Takana, Y. Okuno, and H. Yamasaki: "Numerical Simulation of fluid flow in MHD Power Generation Facility", AIAA Paper, 2003-3486 (2003)

( 4 ) I. Nakamori and T. Ikohagi: "Numerical Investigation of Compressibility effect on Turbulent Coutte Flow Using L.E.S.”, CFD Journal, Vol.8, No.2, VIII-243 (1999)

( 5 ) J.W. Deardorff: "A numerical study of three-dimensional turbulent channel flow”, J. Fluid Mechanics, Vol.1, No.2, pp.453-480 (1970)

( 6 ) D.K. Lilly: "The simulation of three dimensional turbulent flow in two dimensions", NCAR Report (1969)

( 7 ) M. Mitcher and C.H. Kruger: "Partially Ionized gas", John Wiley and Sons, New York (1973)

( 8 ) E. Hinnov and J.G. Hirschberg: "Electron-ion recombination in dense plasmas", Phys. Rev., Vol.125, No.3, pp.795-801 (1962)

( 9 ) S.W. Simpson: "Ionization and recombination rates in argon plasmas", $J$. Phys. D: Appl. Phys., Vol.22, No.8, pp.1161-1167 (1990)

(10) T. Yabe and T. Taoki: "A universal solver for hyperbolic equations by cubicpolynomial interpolation", Comp. Phys. Comun., Vol.66, pp.219-232 (1991)

(11) H.A. Van der Vorst: "Bi-CGSTAB: A fast and smoothly converging variant of Bi-CG for the solution of nonsymmetric linear systems", SIAM J. Sci. Stat. Comput., Vol.13, No.2, pp.631-644 (1992)

(12) A. Liberati, T. Murakami, Y. Okuno, and H. Yamasaki: "Flow behavior in the disk MHD generator of the supersoni closed loop experimental facility for CCMHD power generation", Proc. $15^{\text {th }}$ Int. Conference MHD Energy Conv., Vol.1, pp.113-124, Moscow (2005)

(13) A.H. Shapiro: "The dynamics and thermodynamics of compressible fluid flow", Vol.2, The Ronald Press Company, New York (1956)

(14) N. Harada, T. Ishikawa, N. Kizuka, K. Nakamichi, T. Okamura, and S. Shioda: "Effect of channel shape on the performance of CCMHD generator", Proc. $11^{\text {th }}$ Int. Conf. MHD Energy Conv., Vol.4, pp.1161-1167, Beijing (1992)

(15) T. Suekane, K. Yoshikawa, and S. Kabashima: "Non equilibrium magnetohydrodynamic flow behavior in a closed cycle disk generator", Proc. $11^{\text {th }}$ Int. Conference MHD Energy Conv., Vol.4, pp.1175-1182, Beijing (1992)

\footnotetext{
Alessandro Liberati (Non-member) received the M.E. degree in mechanical engineering from the University of Rome "La Sapienza", Italy, in 2002. He is presently studying for the doctorate degree in energy sciences at the Tokyo Institute of Technology. His research interests include fluid dynamics, closed-cycle MHD power generation and non-equilibrium plasma.
}

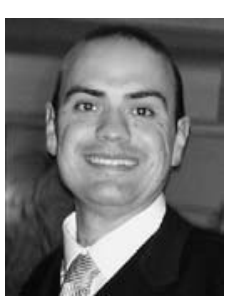


Tomoyuki Murakami (Member) received the B.E. in electrical engi-

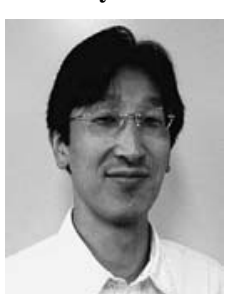
neering from the Tokyo Science University of Chiba, Japan, in 1993 and the M.E. and D.E. degrees in energy sciences from the Tokyo Institute of Technology, Yokohama, Japan, in 1995, and 1997, respectively. $\mathrm{He}$ is presently an Assistamt Professor at the Tokyo Institute of Technology.

Yoshihiro Okuno (Member) received the B.E. in electrical engineer-

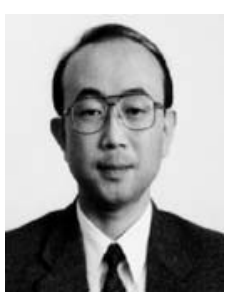
ing from the Kyushu Institute of Technology, Kitakyushu, Japan, and the M.E. and D.E. degrees in energy sciences from the Tokyo Institute of Technology, in 1982, 1984, and 1987, respectively. He is presently a Professor at the Tokyo Institute of Technology.
Hiroyuki Yamasaki (Member) received the B.E. degree from Niigata

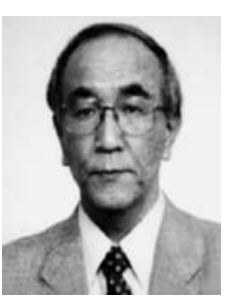
University, Japan, in 1967 and the M.E. and D.E. degrees in energy sciences from the Tokyo Institute of Technology, in 1969 and 1972, respectively. He is presently a Professor at the Tokyo Institute of Technology. 